\title{
Intracorporeal direct measurement for localizing peripheral pulmonary nodules during thoracoscopy
}

\author{
Ming-Wei Kao ${ }^{1,2}$ \\ ${ }^{1}$ Division of Thoracic Surgery, Department of Surgery, E-Da Hospital, Kaohsiung; ${ }^{2}$ School of Medicine for International Students, College of \\ Medicine, I-Shou University, Kaohsiung \\ Correspondence to: Ming-Wei Kao. Division of Thoracic Surgery, Department of Surgery, E-Da Hospital, No. 1, Yida Rd., Yanchao Dist., Kaohsiung. \\ Email: kao.m.wei@gmail.com.
}

Background: Localizing small lung nodules during thoracoscopy is challenging for thoracic surgeons. In this case series, a novel technique for intraoperative localization that combines preoperative computed tomography (CT) with direct measurement during thoracoscopy is described.

Methods: A preoperative CT within two months before surgery was mandatory for precise planning of the resection area. During thoracoscopy, intracorporeal direct measurement (ICDM) for intraoperative localization was undergone if the targeted nodule was non-palpable and non-visualized. According to the location of the targeted nodule, longitudinal, and horizontal landmarks were chosen. The distances between the nodule and these landmarks were obtained from both CT images and intraoperative measurements during thoracoscopy. Based on the measurements, the $\mathrm{x}$-axis and $\mathrm{y}$-axis coordinates of the nodule were calculated and marked on the visceral pleura. A thoracoscopic wedge resection with an adequate margin was performed. From July 2014 to December 2018, ICDM was applied in 27 patients with peripheral pulmonary nodules smaller than $2 \mathrm{~cm}$. Their medical records were reviewed retrospectively to evaluate the feasibility and safety of this technique.

Results: Twenty-six of the twenty-seven nodules were successfully identified (96.3\%). The nodules included 13 primary lung cancers, 5 metastases, and 9 benign lesions. The median nodule size was 7 mm (range, 4-17 mm), and the median distance of the nodule from the visceral pleura was $8.1 \mathrm{~mm}$ (range, 1.0-31.2 $\mathrm{mm}$ ). The median localization time was 24 minutes (range, $8-109 \mathrm{~mm}$ ). Two patients $(7.4 \%$ ) had a prolonged air leak, but there was no procedure-related mortality.

Conclusions: ICDM is an effective and safe method for localizing peripheral lung nodules during thoracoscopy.

Keywords: Video-assisted thoracic surgery; thoracoscopy; computed tomography (CT); localization; pulmonary nodules

Submitted Apr 30, 2019. Accepted for publication Aug 27, 2019.

doi: $10.21037 /$ jtd.2019.10.06

View this article at: http://dx.doi.org/10.21037/jtd.2019.10.06

\section{Introduction}

Thoracoscopic surgery provides similar survival benefits but results in a shorter length of hospital stay as compared to open surgery in early-stage non-small cell lung cancer (1) and is thought to be the mainstay procedure for the excision of small lung nodules. It is, however, challenging for thoracic surgeons to identify these small lesions with the thoracoscopic approach through minimal skin incisions. Several techniques have been introduced to help surgeons to localize the targeted nodule, such as computed tomography (CT)-guided marking methods, electromagnetic navigation bronchoscopy (ENB), intracavitary ultrasound guidance, a hybrid operating theater and three-dimensional (3D) CTsimulation (2-6). These techniques provide remarkable advantages over conventional palpation and inspection 
in localizing the targeted nodule. At the same time, some of these techniques need special instruments or require the participation of experienced interventional specialists, resulting in increased medical costs and patients' waiting time. These techniques are also not always available or feasible. For example, ENB, a hybrid operating theater, and 3D CT-modeling require expensive instruments and special software that may not be available in some hospitals. CTguided marking may not be a valid option if the nodule is near a vessel, the patient has a high risk of pneumothorax or bilateral lung nodules, or no experienced interventional radiologist is available.

CT scans are obtained for nearly all patients who undergo thoracoscopic surgery and are valuable in localizing lung nodules. Consequently, a localizing method that combines CT images with intracorporeal direct measurement (ICDM) to facilitate the intraoperative localization of a targeted pulmonary nodule during thoracoscopy is developed. The aim is to introduce this technique and evaluate its safety and feasibility.

\section{Methods}

\section{Study design and patients}

Between July 2014 and December 2018, the medical records of all consecutive 152 patients with a peripheral lung nodule $\leq 2 \mathrm{~cm}$ hospitalized for thoracoscopic excision were retrospectively reviewed. The patients who underwent ICDM were included for this study. The selection criteria for ICDM were patients who had peripheral lung nodules with the size $\leq 2 \mathrm{~cm}$ and the depth $\leq 3 \mathrm{~cm}$ on CT, and the targeted nodules were not detectable by conventional palpation and inspection during thoracoscopy. All patients had an indication for the surgical excision of the targeted nodules that was either highly suspicious for primary or metastatic malignancy or had a clinically inconclusive diagnosis after an at least 3-month follow-up. Written informed consent was obtained from each patient before the surgery. None of these patients underwent any preoperative localization. This study was approved by the institutional review board of E-Da Hospital (approval number: EMRP108-013).

\section{Surgical technique}

For precise planning of the resection area, a preoperative CT within no more than two months before surgery was mandatory. The patient was placed in the lateral decubitus position under general anesthesia with endotracheal intubation. Single-lung ventilation was achieved by either double-lumen endotracheal tube intubation or single-lumen tube intubation with an endobronchial blocker depending on the evaluation of the anesthesiologist. A $10-\mathrm{mm}$ skin incision was made along the mid-axillary line in the $7^{\text {th }}$ or $8^{\text {th }}$ intercostal space (ICS) for a $10-\mathrm{mm} 30^{\circ}$ scope. Another $30-\mathrm{mm}$ skin incision was made along the anterior axillary line in the $5^{\text {th }}$ or $6^{\text {th }}$ ICS. An inframammary incision was preferred in female patients, if feasible. Carbon dioxide was not routinely insufflated, but only if the lung collapse was not ideal. The operator stood at the patient's ventral side and performed the surgery via the anterior working port. The assistant stood at the dorsal side of the patient to hold the scope. First, the operator aimed to detect the nodule by visual inspection and palpation using fingers or instruments via the working port. When the nodule could be identified, wedge resection was performed directly.

In cases where the nodule was not detectable with this method, ICDM was performed. The steps were as follows:

\section{Choosing the horizontal landmark and calculating the y-axis coordinate}

Because of their rigidity and coverage by the mediastinal pleura, the position of the hilar structures remains almost unchanged even with complete lung collapse, and they are therefore suitable to guide nodule location. For the left lung, the level between the sub-aortic arch (sub-AA) and inferior pulmonary vein (IPV) was fixed (Figure 1A). For the right lung, the level between the azygos vein (AV) and IPV was fixed (Figure 1B). These landmarks divided the lung into four horizontal zones: from the apex to sub-AA/ $\mathrm{AV}$, from sub-AA/AV to the sixth bronchus (B6), from B6 to the IPV, and from the IPV to the base. Within these four zones, the first and fourth were movable while the second and third were fixed. If the nodule was located in the second or third part, its y-axis location was calculated from the nearest landmark on the CT, for example, $1 \mathrm{~cm}$ above the IPV. If the nodule was located in the first or fourth part, the $y$-axis location was calculated in proportion to the distance between the IPV and base after releasing the pulmonary ligaments. For example, if the nodule was located $3 \mathrm{~cm}$ below the IPV and $1 \mathrm{~cm}$ above the base on the CT, its y-axis coordinate was at a ratio of 3:1 from the measured distance between the IPV and base during thoracoscopy. If there was a complete fissure, this could be used as a landmark, and a nodule located in zone 3 of the right upper lobe (RUL) 

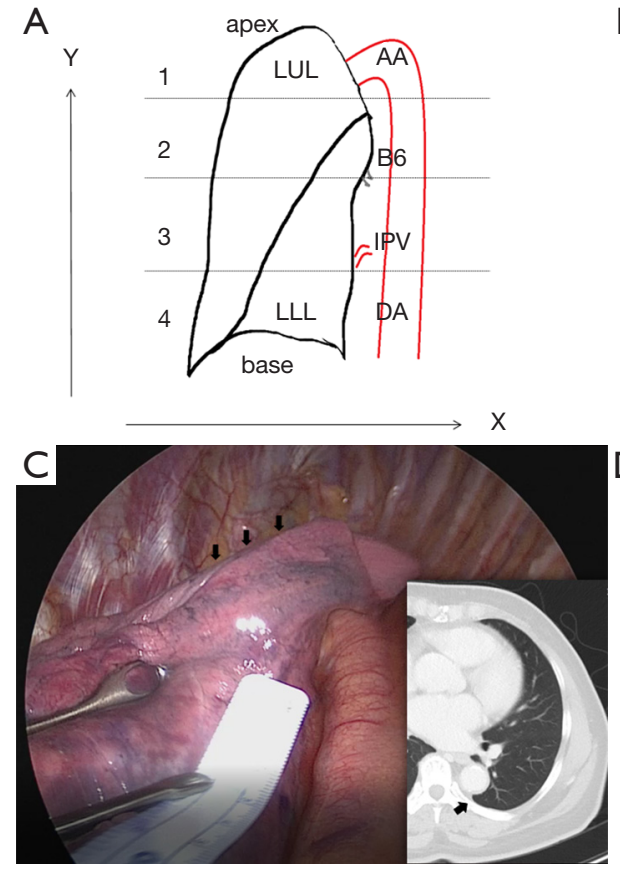

B

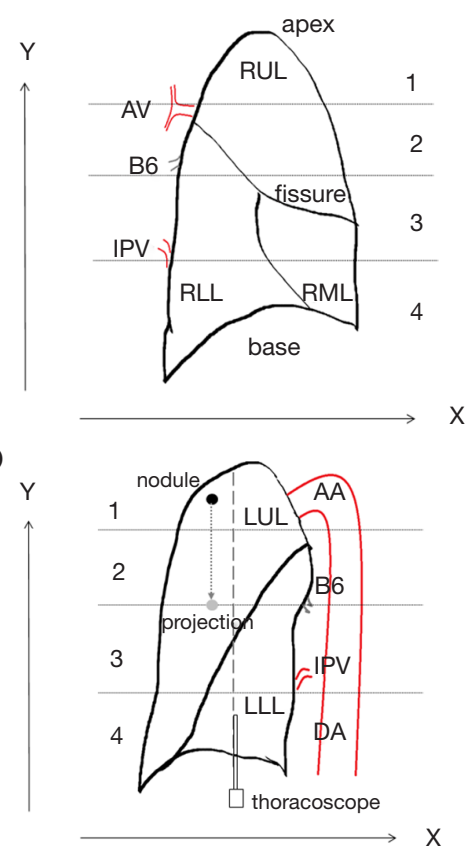

Figure 1 Illustration of landmarks for intracorporeal direct measurement. (A) Left lung; (B) right lung; (C) convexity of the aorta (arrow); (D) the projection of the nodule in zone 1 (black) to zone 2 (gray) and the mid-axillary line (dashed) helps to establish the longitudinal landmark. LUL, left upper lobe; LLL, left lower lobe; RUL, right upper lobe; RML, right middle lobe; RLL, right lower lobe; AA, aortic arch; DA, descending aorta; B6, the bronchus of the sixth segment; IPV, inferior pulmonary vein; AV, azygos vein.

was managed in proportion to the distances between the fissure and the hilar structures. It needs to be emphasized that the distance on the visceral pleura should be obtained, especially for the lesions located in RUL and left upper division, because the pleura line was a curve not a straight line.

\section{Choosing the longitudinal landmark and obtaining the} distances on CT

For the left lung, the descending thoracic aorta (DA) forms a convexity on the lung surface, which provides an useful landmark (Figure 1C). For the right lung, the mediastinum and the fissure (lobar edge) were used as landmarks. The horizontal distances on the visceral pleura between the fissure, the surface projection of the target nodule, and the DA or mediastinum were obtained from the preoperative CT. For lesions located in the first and fourth zone, it was sometimes challenging to choose a longitudinal landmark in this way. In this situation, there were two alternative ways to establish the longitudinal landmark: making a projection to the second or third zone, or using the mid-axillary line, where the camera port was situated (Figure 1D).
Measuring the distance between landmarks on the visceral pleura and calculating the $x$-axis coordinate Under thoracoscopic inspection, a sterile paper ruler (Aspen Surgical, Caledonia, MI, USA) was placed on the lung surface at the level of the $y$-axis coordinate to measure the distance between the fissure and the DA or mediastinum, the chosen landmark (Figure $2 A, B$ ). In proportion to the ratio of the $\mathrm{CT}$ distance to this measured distance, the $\mathrm{x}$-axis coordinate of the projection point was obtained and marked on the pleura (Figure 2C,D). Depending on the depth of the nodule, wedge resection with adequate margins was then performed using an endostapler (Figure $2 E, F$ ). The video demonstration of this technique was shown in Figures 3,4.

\section{Data collection and analysis}

From the institutional prospectively maintained medical database, the demographic data and surgical outcomes were retrieved and reviewed. The descriptive statistics are presented as number (percentage) for categorical data and as median with interquartile range and range for continuous data, and were carried out with IBM SPSS Statistics for 

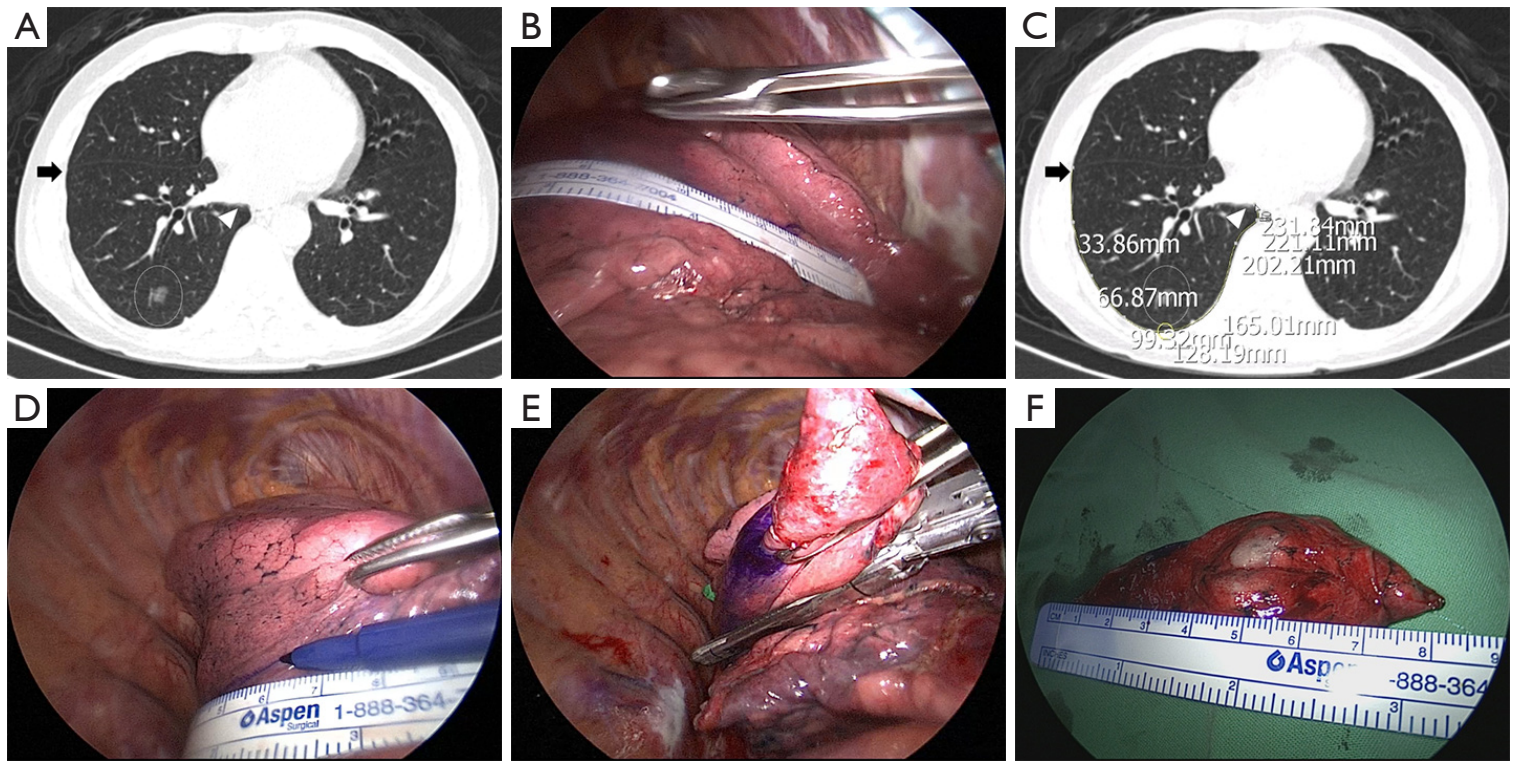

Figure 2 Steps of localization by intracorporeal direction measurement. (A) On preoperative computed tomography (CT) images, choose the mediastinal landmark for the targeted nodule (dotted circle). In this case, right inferior pulmonary vein (IPV, arrowhead) and the lobar fissure (black arrow) are used as the landmark; (B) use a sterile paper ruler to obtain the distance between the horizontal landmarks: the fissure and right IPV; (C) obtain the distances between the fissure (black arrow), the targeted nodule (dotted circle), and right IPV (arrowhead) on the preoperative CT images; (D) mark the lesion on the visceral pleura after calculating the distance; (E) wedge resection with adequate margin is performed; $(\mathrm{F})$ the targeted nodule is successfully removed.

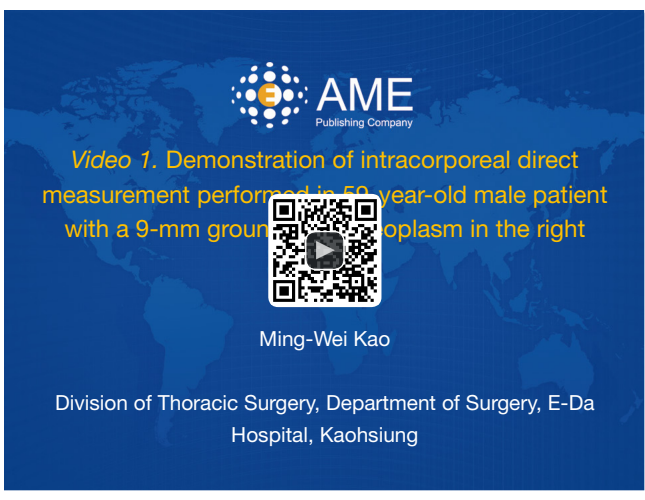

Figure 3 Demonstration of intracorporeal direct measurement performed in 59-year-old male patient with a 9-mm ground-glass neoplasm in the right lower lobe (7).

Available online: http://www.asvide.com/watch/32995

Windows, version 22 (IBM Corp., Armonk, NY, USA).

\section{Results}

ICDM was performed in 27 patients with peripheral

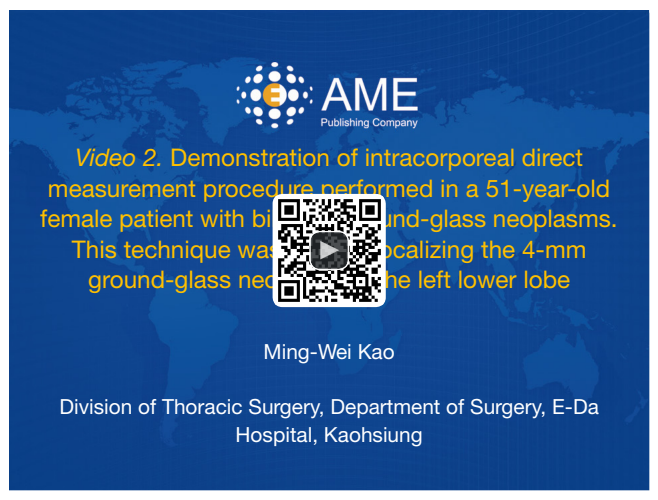

Figure 4 Demonstration of intracorporeal direct measurement procedure performed in a 51-year-old female patient with bilateral ground-glass neoplasms. This technique was used in localizing the 4-mm ground-glass neoplasm in the left lower lobe (8). Available online: http://www.asvide.com/watch/32996

pulmonary nodules $\leq 2 \mathrm{~cm}$. Among these 27 patients, ground-glass neoplasms (GGNs), defined as lesions with consolidation-to-tumor ratio $\leq 0.5$, were found on the preoperative CT in 20 patients $(74 \%)$. Patient characteristics are summarized in Table 1 . The median 
Table 1 Clinical features in 27 cases undergoing thoracoscopic resection of peripheral pulmonary nodules after localization by direct measurement

\begin{tabular}{lc}
\hline Variables & Values (N=27) \\
\hline Gender & $18(67 \%)$ \\
Female & $9(33 \%)$ \\
Male & $59(15)[36-72]$ \\
Age (years) & $24.3(5.6)[18.1-31.1]$ \\
Body mass index $\left(\mathrm{kg} / \mathrm{m}^{2}\right)$ & $7(5.1)[4-17]$ \\
Nodule size $(\mathrm{mm})$ & $8.1(10.7)[1.0-31.2]$ \\
Depth on CT (mm) & $20(74 \%)$ \\
GGN (CTR $\leq 0.5)$ & \\
Nodule location & $1(4 \%)$ \\
Left upper lobe & $6(22 \%)$ \\
Left lower lobe & $10(37 \%)$ \\
Right upper lobe & $0(0 \%)$ \\
Right middle lobe & $10(37 \%)$ \\
Right lower lobe &
\end{tabular}

Categorical data are presented as number (percentage) and continuous data presented as median (interquartile range) [range]. CT, computed tomography; GGN, ground-glass neoplasm; CTR, consolidation-to-tumor ratio.

localization time was 24 minutes. Two patients (7.4\%) had severe pleural adhesions and needed extensive adhesiolysis, resulting in a longer localization time (80 and 109 minutes) as compared to the other patients and prolonged air leaks. Three patients underwent bilateral thoracoscopic excisions of multiple pulmonary nodules $(11.1 \%)$. Twenty-six nodules (96.3\%) were successfully localized by ICDM. One nodule could not be identified with the method $(3.7 \%)$ but was successfully removed by segmentectomy. The surgical outcomes are shown in Table 2.

\section{Discussion}

In the era of minimally invasive surgery, it is critical for thoracic surgeons to localize a small targeted nodule via a small incision. In the current practice of preoperative localization, CT-guided marking techniques are widely used, for example, the placement of a hookwire $(9,10)$ or injection of detectable agents (11-14). However, an accidental penetration of the visceral pleura during these procedures may cause complications, some
Table 2 Surgical outcomes of 27 cases undergoing thoracoscopic resection of peripheral pulmonary nodules after localization by direct measurement

\begin{tabular}{lc}
\hline Variables & Values $(\mathrm{N}=27)$ \\
\hline Successful localization & $26(96.3 \%)$ \\
Localization time (minutes) & $24(53)[8-109]$ \\
Bilateral surgery & $3(11.1 \%)$ \\
Operative method following localization & \\
Wedge resection & $19(70.4 \%)$ \\
Segmentectomy & $2(7.4 \%)^{\star}$ \\
Lobectomy & $6(22.2 \%)$ \\
Tube drainage (days) & $2(1.3)[0-27]$ \\
Hospital stay (days) & $5(4)[2-28]$ \\
Complication & $2(7.4 \%)$ \\
Prolonged air leak & 0 \\
Mortality & \\
Histology & $13(48.1 \%)$ \\
Malignant, primary lung & $5(18.5 \%)$ \\
Malignant, metastasis & $3(11.1 \%)$ \\
Benign, infectious & $6(22.2 \%)$ \\
\hline Catenign, non-infectious & $(p e r c e n t a g)$ and
\end{tabular}

Categorical data are presented as number (percentage) and continuous data presented as median (interquartile range) [range]. *, including the case in which the intracorporeal direct measurement failed to localized the nodule, that consecutively was successfully removed by segmentectomy.

of which are potentially disastrous, such as hematoma, hemothorax, pneumothorax, air embolism, or pleural seeding of the tumor (15-19). Zaman et al. (20) reviewed the literature and reported the success rate of the hookwire technique to differ between hospitals, ranging from $58 \%$ to $97.6 \%$, and the failures were mainly caused by wire dislodgement. Ciriaco et al. (10,21), reporting on their experience, suggested that preoperative CT-guided hook-wire localization is a useful technique to identify pulmonary nodules during thoracoscopy when performed by experienced hands. Dye injection has been considered to be of equivalent accuracy but has less severe complications compared to hook-wire placement (22). However, a failure rate of $13 \%$ has been reported, part of which resulted from an excessive volume of dye injected, an error that might be minimized in expert hands $(12,23,24)$. For patients 
with a high risk of pneumothorax and patients with lesions located near vessels or close to the scapula and ribs, the interventional radiologist with limited experience is unlikely to suggest CT-guided marking. In an article discussing the most appropriate procedure for localizing small pulmonary nodules, Kato et al. (25) and colleagues emphasized that the surgeon should be familiar with the anatomical structures and make the planning in person because of the surgeon's unique view during surgery. The thoracic surgeon has to find the alternative method to localize the targeted nodule in addition to the conventional visual inspection and digital palpation. Navigational bronchoscopy and hybrid operating rooms are advanced intraoperative techniques to localize the targeted nodule and could be performed by thoracic surgeons personally in the operating theater $(3,5,26,27)$, but these techniques require special instruments and increase patients' medical expense (28) and are therefore unlikely to be available in many institutions.

ICDM is a non-invasive procedure for the localization of a nodule during thoracoscopy based on a combination of preoperative CT images, intraoperative measurements, and simple mathematic calculations. No special instruments are required. It is designed to assist the operator in identifying targeted nodules that could not be detected by inspection and palpation, and when preoperative localization is not available. Especially for patients with bilateral lung lesions, preoperative CT-guided hook-wire or dye marking is concerning due to the possibility of bilateral pneumothorax, wire migration, and dye diffusion with time, which will make the nodule difficult to identify. For these patients, ICDM is an alternative method for nodule localization.

ICDM is suggested in cases that the distance between the targeted nodule and the visceral pleura is less than $3 \mathrm{~cm}$. In this report, there was only one patient who had a nodule locating $31.2 \mathrm{~mm}$ deep from the visceral pleura. The reason is that the severe adhesion in his pleura cavity made segmentectomy much more difficult than ICDM to localize and remove the nodule. Performing segmentectomy is another useful method to localize pulmonary nodules. Kato et al. (6) showed that thoracoscopic anatomical segmentectomy using 3D CT simulation is effective in removing non-palpable and non-visible lung nodules. In my institute's experience, segmentectomy was excellent to remove centrally located pulmonary nodules but took much more time than ICDM to remove peripheral nodules [median time: 135.5 (IQR: 60) minutes versus 56.5 (IQR: 78.8) minutes; Mann-Whitney $\mathrm{U}$ test $\mathrm{P}=0.001$. Unpublished data].
In this series, $96.3 \%$ of nodules were successfully identified and removed. The case in which ICDM failed occurred during the early phase of applying this method $\left(6^{\text {th }}\right.$ patient). The nodule was located in the $3^{\text {rd }}$ segment of RUL with an incomplete fissure and $17.7 \mathrm{~mm}$ deep from the visceral pleura. I failed to remove it because I did not separate the fissure enough for an adequate resection margin. After applying the endostapler, it did not reach deep enough to include the nodule that consecutively was successfully removed by segmental resection.

In my experience, ICDM has two limitations. First, it is challenging to perform in cases of severe pleural adhesions, because it needs at least two completely exposed landmarks as the $\mathrm{x}$-axis and $\mathrm{y}$-axis coordinates. If a comprehensive adhesiolysis is necessary to establish these landmarks, the localization time and probably also the air leak are prolonged. Second, ICDM only provides an estimated surface marking, and the operator must evaluate the nodule depth based on the preoperative CT images to decide on the resection margin. In other words, ICDM may not be beneficial for a centrally located lesion, which may be removed by segmental resection instead. Similar to the case described above, segmentectomy is strongly recommended for rescue.

This is a small-sized retrospective study based on a single surgeon's experience, introducing a selection bias. ICDM requires validation by other surgeons in the future to improve the technique and establish the optimal indications. The cost-effectiveness of ICDM compared with other localization techniques may also need to be investigated.

\section{Conclusions}

In conclusion, for peripheral small pulmonary nodules which are not detectable by the conventional palpation and inspection, ICDM is a feasible and safe method for intraoperative localization.

\section{Acknowledgments}

None.

\section{Footnote}

Conflicts of Interest: The author has no conflicts of interest to declare.

Ethical Statement: The author is accountable for all 
aspects of the work in ensuring that questions related to the accuracy or integrity of any part of the work are appropriately investigated and resolved. The study was approved by the institutional review board of E-Da Hospital (approval number: EMRP-108-013) and written informed consent was obtained from all patients.

\section{References}

1. Yang HX, Woo KM, Sima CS, et al. Long-term Survival Based on the Surgical Approach to Lobectomy For Clinical Stage I Nonsmall Cell Lung Cancer: Comparison of Robotic, Video-assisted Thoracic Surgery, and Thoracotomy Lobectomy. Ann Surg 2017;265:431-7.

2. Lin MW, Chen JS. Image-guided techniques for localizing pulmonary nodules in thoracoscopic surgery. J Thorac Dis 2016;8:S749-55.

3. Goud A, Dahagam C, Breen DP, et al. Role of electromagnetic navigational bronchoscopy in pulmonary nodule management. J Thorac Dis 2016;8:S501-8.

4. Khereba M, Ferraro P, Duranceau A, et al. Thoracoscopic localization of intraparenchymal pulmonary nodules using direct intracavitary thoracoscopic ultrasonography prevents conversion of VATS procedures to thoracotomy in selected patients. J Thorac Cardiovasc Surg 2012;144:1160-5.

5. Hsieh CP, Hsieh MJ, Fang HY, et al. Imaging-guided thoracoscopic resection of a ground-glass opacity lesion in a hybrid operating room equipped with a robotic C-arm CT system. J Thorac Dis 2017;9:E416-9.

6. Kato H, Oizumi H, Suzuki J, et al. Thoracoscopic anatomical lung segmentectomy using $3 \mathrm{D}$ computed tomography simulation without tumour markings for nonpalpable and non-visualized small lung nodules. Interact Cardiovasc Thorac Surg 2017;25:434-41.

7. Kao MW. Demonstration of intracorporeal direct measurement performed in 59-year-old male patient with a 9-mm ground-glass neoplasm in the right lower lobe. Asvide 2019;6:310. Available online: http://www.asvide. com/watch/32995

8. Kao MW. Demonstration of intracorporeal direct measurement procedure performed in a 51-year-old female patient with bilateral ground-glass neoplasms. This technique was used in localizing the 4-mm groundglass neoplasm in the left lower lobe. Asvide 2019;6:311. Available online: http://www.asvide.com/watch/32996

9. Mack MJ, Gordon MJ, Postma TW, et al. Percutaneous localization of pulmonary nodules for thoracoscopic lung resection. Ann Thorac Surg 1992;53:1123-4.
10. Ciriaco P, Negri G, Puglisi A, et al. Video-assisted thoracoscopic surgery for pulmonary nodules: rationale for preoperative computed tomography-guided hookwire localization. Eur J Cardiothorac Surg 2004;25:429-33.

11. Lenglinger FX, Schwarz CD, Artmann W. Localization of pulmonary nodules before thoracoscopic surgery: value of percutaneous staining with methylene blue. AJR Am J Roentgenol 1994;163:297-300.

12. Lin MW, Tseng YH, Lee YF, et al. Computed tomography-guided patent blue vital dye localization of pulmonary nodules in uniportal thoracoscopy. J Thorac Cardiovasc Surg 2016;152:535-44.e2.

13. Finley RJ, Mayo JR, Grant K, et al. Preoperative computed tomography-guided microcoil localization of small peripheral pulmonary nodules: a prospective randomized controlled trial. J Thorac Cardiovasc Surg 2015;149:26-31.

14. Kim YD, Jeong YJ, I H, et al. Localization of pulmonary nodules with lipiodol prior to thoracoscopic surgery. Acta Radiol 2011;52:64-9.

15. Wu CC, Maher MM, Shepard JA. Complications of CT-guided percutaneous needle biopsy of the chest: prevention and management. AJR Am J Roentgenol 2011;196:W678-82.

16. Horan TA, Pinheiro PM, Araujo LM, et al. Massive gas embolism during pulmonary nodule hook wire localization. Ann Thorac Surg 2002;73:1647-9.

17. Wiener RS, Schwartz LM, Woloshin S, et al. Populationbased risk for complications after transthoracic needle lung biopsy of a pulmonary nodule: an analysis of discharge records. Ann Intern Med 2011;155:137-44.

18. Inoue $\mathrm{M}$, Honda $\mathrm{O}$, Tomiyama $\mathrm{N}$, et al. Risk of pleural recurrence after computed tomographic-guided percutaneous needle biopsy in stage I lung cancer patients. Ann Thorac Surg 2011;91:1066-71.

19. Shyamala K, Girish HC, Murgod S. Risk of tumor cell seeding through biopsy and aspiration cytology. J Int Soc Prev Community Dent 2014;4:5-11.

20. Zaman M, Bilal H, Woo CY, et al. In patients undergoing video-assisted thoracoscopic surgery excision, what is the best way to locate a subcentimetre solitary pulmonary nodule in order to achieve successful excision? Interact Cardiovasc Thorac Surg 2012;15:266-72.

21. Ciriaco P, Muriana P, Negri G. Pulmonary nodules and mini-invasive lung resection: do we have the right "tool" for their intraoperative localization? J Thorac Dis 2017;9:4216-8.

22. Kleedehn M, Kim DH, Lee FT, et al. Preoperative 
Pulmonary Nodule Localization: A Comparison of Methylene Blue and Hookwire Techniques. AJR Am J Roentgenol 2016;207:1334-9.

23. Vandoni RE, Cuttat JF, Wicky S, et al. CT-guided methylene-blue labelling before thoracoscopic resection of pulmonary nodules. Eur J Cardiothorac Surg 1998;14:265-70.

24. Tao G, Jingying Y, Tan G, et al. A novel CT-guided technique using medical adhesive for localization of small pulmonary ground-glass nodules and mixed ground-glass nodules $(</=20 \mathrm{~mm}$ ) before video-assisted thoracoscopic surgery. Diagn Interv Radiol 2018;24:209-12.

25. Kato H, Oizumi H, Suzuki J, et al. What is the most appropriate procedure for intraoperative localization of

Cite this article as: Kao MW. Intracorporeal direct measurement for localizing peripheral pulmonary nodules during thoracoscopy. J Thorac Dis 2019;11(10):4119-4126. doi: $10.21037 /$ jtd.2019.10.06 small pulmonary nodules? J Thorac Dis 2018;10:E155-7.

26. Seijo LM. Electromagnetic navigation bronchoscopy: clinical utility in the diagnosis of lung cancer. Lung Cancer (Auckl) 2016;7:111-8.

27. Zhao ZR, Lau RW, Ng CS. Hybrid theatre and alternative localization techniques in conventional and singleport video-assisted thoracoscopic surgery. J Thorac Dis 2016;8:S319-27.

28. Dale CR, Madtes DK, Fan VS, et al. Navigational bronchoscopy with biopsy versus computed tomographyguided biopsy for the diagnosis of a solitary pulmonary nodule: a cost-consequences analysis. J Bronchology Interv Pulmonol 2012;19:294-303. 